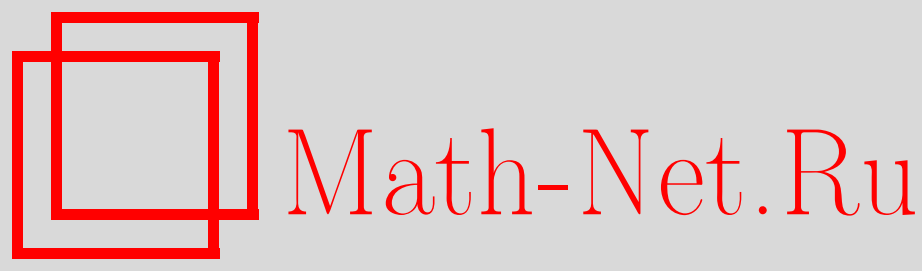

П. П. Костробий, А. В. Визнович, Б. Б. Маркив, М. В. Токарчук, Обобщенные кинетические уравнения для плотных газов и жидкостей в методе неравновесного статистического оператора Зубарева и статистике Реньи, ТМФ, 2015, том 184, номер 1, 145-159

DOI: https://doi.org/10.4213/tmf8811

Использование Общероссийского математического портала Math-Net.Ru подразумевает, что вы прочитали и согласны с пользовательским соглашением http://www.mathnet.ru/rus/agreement

Параметры загрузки:

IP : 54.198 .55 .26

26 апреля 2023 г., $11: 38: 58$

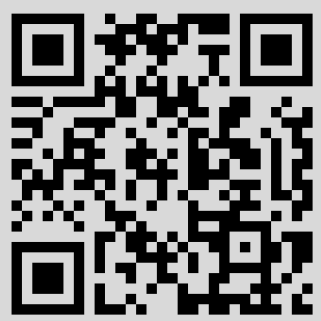




\title{
ОБОБЩЕННЫЕ КИНЕТИЧЕСКИЕ УРАВНЕНИЯ ДЛЯ ПЛОТНЫХ ГАЗОВ И ЖИДКОСТЕЙ В МЕТОДЕ НЕРАВНОВЕСНОГО СТАТИСТИЧЕСКОГО ОПЕРАТОРА ЗУБАРЕВА И СТАТИСТИКЕ РЕНЬИ
}

\begin{abstract}
Для описания кинетических процессов в газах и жидкостях, далеких от равновесия, на основании метода неравновесного статистического оператора Зубарева и принципа максимума энтропии Реньи получены неравновесный статистический оператор и обобщенные кинетические уравнения для неравновесных одночастичной и двухчастичной функций распределения. Рассмотрено $q$-обобщение уравнения Лиувилля и методом неравновесного статистического оператора получено его общее решение.
\end{abstract}

Ключевые слова: энтропия Реньи, неравновесный статистический оператор, обобщенные кинетические уравнения.

DOI: $10.4213 / \operatorname{tmf} 8811$

\section{1. ВВЕДЕНИЕ}

Изучение нелинейных флуктуаций в плотных газах, плазме, а также в процессах турбулентности, динамике фазовых переходов, химических реакциях, процессах самоорганизации остается актуальным в статистической теории неравновесных процессов [1]-[18] как на кинетическом, так и на гидродинамическом уровне описания. Состояния таких систем далеки от равновесия. Поэтому важно, с одной стороны, изучать процессы, которые приводят к появлению устойчивых состояний с характерными временами жизни, с другой стороны, исследовать процессы релаксации системы к неравновесным состояниям, которые уже хорошо изучены (например, в случае плотных газов и жидкостей процессы релаксации к состояниям, которые описываются в рамках теории молекулярной гидродинамики [19]-[22]). Важно отметить, что еще одной особенностью неравновесных явлений в плотных газах, жидкостях,

*Национальный университет "Львовская политехника", Львов, Украина

${ }^{\dagger}$ Институт физики конденсированных систем НАН Украины, Львов, Украина. E-mail: markiv@icmp.lviv.ua 
плотной плазме (а также в пылевой плазме) является то, что кинетические и гидродинамические процессы следует рассматривать согласованно [23]-[27]. В таких исследованиях используются различные статистические подходы на основе энтропии Гиббса-Шеннона [28]-[32] и ее обобщений (энтропии Цаллиса [33], Реньи [34], [35], Шармы-Миттала [36], [37]), а также суперстатистики [38], [39].

Энтропия Цаллиса широко используется в различных направлениях неэкстенсивной статистической механики (см. работы [40]-[42] и ссылки в них), например при исследовании явлений субдиффузии [43], [44] и турбулентности [45], [46], коэффициентов переноса в газах и плазме [47], а также квантовых диссипативных систем [48]. В рамках формализма Цаллиса исследовались флуктуации энергии [49], кинетика неравновесной плазмы [50], проблемы самогравитирующих [51] и сложных систем [52], [53]. В работах [54]-[56] статистика Цаллиса применялась для описания химических реакций, в частности нелинейные уравнения реакционно-диффузионных процессов были получены в работе [54]. Несмотря на широкое применение энтропии Цаллиса как обобщения энтропии Гиббса-Шеннона, энтропия Реньи также представляет большой интерес [18], [43], [57]-[64]. В частности, в этом случае индекс $q$ можно связать с теплоемкостью системы [60]. Важно отметить работы Луцци и др. [65], [66], в которых метод неравновесного статистического оператора и энтропия Реньи используются для описания систем, далеких от равновесия. В частности, неравновесное $q$-зависимое распределение Реньи, а также обобщенные функции распределения бозонов и фермионов были получены в работе [65], в которой в этом подходе были также описаны эксперименты по аномальной люминесценции в нанометровых квантовых точках в полупроводниковых гетероструктурах. Статистический подход для описания фрактальных физико-химических систем на основе нефиковских диффузионных процессов был предложен в работе [66], где проводились исследования аномальной диффузии в фракталоподобных электродах микробатарей. Неэкстенсивный подход [67], как и другие подходы [68], [69], которые приводят к уравнению Линдблада, использовались для описания декогеренции в квантовой механике. Работы [17], [70], [71] посвящены исследованиям нелинейной кинетики на основе уравнений Крамерса, Больцмана и Фоккера-Планка в рамках обобщенной статистики.

В настоящей работе для описания неравновесных процессов в плотных газах и жидкостях мы используем энтропию Реньи, которая зависит от параметра $q(0<$ $q \leqslant 1)$ и совпадает с энтропией Гиббса-Шеннона при $q=1$. В работе [18] на основании метода неравновесного статистического оператора Зубарева [28], [30], [31] и принципа максимума энтропии Реньи были получены неравновесный статистический оператор и обобщенные уравнения переноса для параметров сокращенного описания неравновесных процессов в экстенсивной статистической механике. В настоящей работе мы применяем данный подход к описанию кинетических процессов в плотных газах и жидкостях, далеких от равновесия, когда неравновесные однои двухчастичная функции распределения выбраны в качестве параметров сокращенного описания.

В разделе 2 мы формулируем метод неравновесного статистического оператора Зубарева в статистике Реньи. В разделе 3 на его основе получены обобщенные кинетические уравнения для одно- и двухчастичной функций распределения в статистике Реньи. В разделе 4 мы рассматриваем q-обобщение уравнения Лиувилля, получаем его решение с помощью метода неравновесного статистического оператора и обсуждаем его применение. 


\section{2. МЕТОД НЕРАВНОВЕСНОГО СТАТИСТИЧЕСКОГО ОПЕРАТОРА ЗУБАРЕВА В СТАТИСТИКЕ РЕНЬИ}

В методе неравновесного статистического оператора Зубарева, когда параметры сокращенного описания $\left\langle\widehat{P}_{n}\right\rangle^{t}$ выбраны согласно Боголюбову, неравновесный статистический оператор системы $\varrho\left(x^{N} ; t\right)=\varrho\left(x_{1}, \ldots, x_{N} ; t\right)$ может быть найден в общем виде с учетом проецирования как решение уравнения Лиувилля [28], [30], [31]:

$$
\varrho\left(x^{N} ; t\right)=\varrho_{\text {rel }}\left(x^{N} ; t\right)-\int_{-\infty}^{t} e^{\varepsilon\left(t^{\prime}-t\right)} T\left(t, t^{\prime}\right)\left[1-P_{\text {rel }}\left(t^{\prime}\right)\right] i L_{N} \varrho_{\text {rel }}\left(x^{N} ; t^{\prime}\right) d t^{\prime},
$$

где

$$
T\left(t, t^{\prime}\right)=\exp _{+}\left\{-\int_{t^{\prime}}^{t}\left[1-P_{\text {rel }}\left(t^{\prime}\right)\right] i L_{N} d t^{\prime}\right\}
$$

- оператор эволюции с учетом проецирования, $\exp _{+}-$упорядоченная экспонента, $i L_{N}$ - оператор Лиувилля системы взаимодействующих частиц, который в классическом случае имеет следующую форму:

$$
i L_{N}=\sum_{j=1}^{N} \frac{\vec{p}_{j}}{m} \cdot \frac{\partial}{\partial \vec{r}_{j}}-\frac{1}{2} \sum_{\substack{l, j=1 \\ l \neq j}}^{N} \frac{\partial}{\partial \vec{r}_{j}} \Phi\left(r_{l j}\right)\left(\frac{\partial}{\partial \vec{p}_{j}}-\frac{\partial}{\partial \vec{p}_{l}}\right)
$$

Здесь использованы следующие обозначения: $x_{j}=\left\{\vec{p}_{j}, \vec{r}_{j}\right\}$ - фазовые переменные $j$-й частицы, $\Phi\left(r_{l j}\right)$ - энергия взаимодействия двух частиц, $\vec{p}_{j}$ - импульс $j$-й частицы, $m$ - ее масса, $r_{l j}=\left|\vec{r}_{l}-\vec{r}_{j}\right|$ - расстояние между парой взаимодействующих частиц. $P_{\text {rel }}\left(t^{\prime}\right)$ - обобщенный проекционный оператор Кавасаки-Гантона, структура которого зависит от формы релевантного статистического оператора:

$$
P_{\text {rel }} \varrho^{\prime}=\left(\varrho_{\text {rel }}(t)-\sum_{n} \frac{\delta \varrho_{\text {rel }}(t)}{\delta \widehat{P}_{n}^{t}}\left\langle\widehat{P}_{n}\right\rangle^{t}\right) \int \varrho^{\prime} d \Gamma_{N}+\sum_{n} \frac{\delta \varrho_{\text {rel }}(t)}{\delta\left\langle\widehat{P}_{n}\right\rangle^{t}} \int \widehat{P}_{n} \varrho^{\prime} d \Gamma_{N},
$$

где $\varrho_{\text {rel }}\left(x^{N} ; t\right)$ - релевантный статистический оператор, который в начальный момент времени равен $\varrho\left(x^{N} ; t\right)$. Используя метод Лагранжа, будем искать $\varrho_{\text {rel }}\left(x^{N} ; t\right)$ исходя из условия максимума функционала энтропии Реньи

$$
S_{\mathrm{R}}(\varrho)=\frac{1}{1-q} \ln \int \varrho^{q}(t) d \Gamma_{N}
$$

при фиксированных параметрах сокращенного описания и с учетом условия нормировки [18]. Соответствующий функционал имеет следующий вид:

$$
L_{\mathrm{R}}(\varrho)=\frac{1}{1-q} \ln \int \varrho^{q}(t) d \Gamma_{N}-\alpha \int \varrho(t) d \Gamma_{N}-\sum_{n} F_{n}(t) \int \widehat{P}_{n} \varrho(t) d \Gamma_{N},
$$

где $F_{n}(t)$ - множители Лагранжа. Приравнивая к нулю функциональную производную $\delta L_{\mathrm{R}}(\varrho) / \delta \varrho=0$ и определяя параметр

$$
\alpha=\frac{q}{1-q}-\sum_{n} F_{n}(t)\left\langle\widehat{P}_{n}\right\rangle^{t},
$$


мы получим релевантный статистический оператор в следующем виде:

$$
\varrho_{\text {rel }}(t)=\frac{1}{Z_{\mathrm{R}}(t)}\left[1-\frac{q-1}{q} \sum_{n} F_{n}(t) \delta \widehat{P}_{n}\right]^{1 /(q-1)},
$$

где

$$
Z_{\mathrm{R}}(t)=\int d \Gamma_{N}\left[1-\frac{q-1}{q} \sum_{n} F_{n}(t) \delta \widehat{P}_{n}\right]^{1 /(q-1)}
$$

- статистическая сумма, и $\delta \widehat{P}_{n}=\widehat{P}_{n}-\left\langle\widehat{P}_{n}\right\rangle^{t}$. Множители Лагранжа $F_{n}(t)$ определяются из условий самосогласования:

$$
\left\langle\widehat{P}_{n}\right\rangle^{t}=\left\langle\widehat{P}_{n}\right\rangle_{\text {rel }}^{t}
$$

Для того чтобы найти обобщенный проекционный оператор Кавасаки-Гантона (4), релевантный статистический оператор (7) удобно представить в следующем виде:

$$
\varrho_{\text {rel }}(t)=\frac{1}{Z_{\mathrm{R}}^{*}}\left(1-\frac{q-1}{q} \sum_{n} F_{n}^{*}(t) \widehat{P}_{n}\right)^{1 /(q-1)}
$$

со статистической суммой

$$
Z_{\mathrm{R}}^{*}(t)=\int d \Gamma_{N}\left(1-\frac{q-1}{q} \sum_{n} F_{n}^{*}(t) \widehat{P}_{n}\right)^{1 /(q-1)}
$$

и перенормированными множителями

$$
F_{n}^{*}(t)=F_{n}(t)\left(1+\frac{q-1}{q} \sum_{l} F_{l}(t)\left\langle\widehat{P}_{l}\right\rangle^{t}\right)^{-1}
$$

Для того чтобы найти неравновесный статистический оператор (1), необходимо раскрыть явную структуру обобщенного проекционного оператора Кавасаки-Гантона (4). Для этого необходимо рассчитать вариационную производную от релевантного статистического оператора $\delta \rho_{\text {rel }}(t) / \delta\left\langle\widehat{P}_{m}\right\rangle^{t}$, а следовательно, и вариационную производную $\delta F_{n}^{*}(t) / \delta\left\langle\widehat{P}_{m}\right\rangle^{t}$, которую можно рассчитать как $\left[\delta\left\langle\widehat{P}_{m}\right\rangle^{t} / \delta F_{n}^{*}(t)\right]^{-1}$. Тогда действие операторов $P_{\mathrm{rel}}(t) i L_{N}$ на релевантный статистический оператор можно представить в виде

$$
P_{\text {rel }}(t) i L_{N} \varrho_{\text {rel }}(t)=P_{\text {rel }}(t) A(t) \varrho_{\text {rel }}(t)=[P(t) A(t)] \varrho_{\text {rel }}(t),
$$

где мы ввели обобщенный проекционный оператор, действующий на динамические переменные:

$$
P(t) \cdots=\sum_{m, n}\left\langle\ldots \widehat{P}_{m}\right\rangle_{\mathrm{rel}}\left\langle\widehat{P}_{m} \delta\left\{[q \psi(t)]^{-1} \widehat{P}_{n}\right\}\right\rangle_{\mathrm{rel}}^{-1} \delta\left\{[q \psi(t)]^{-1} \widehat{P}_{n}\right\}
$$

где

$$
\psi(t)=1-\frac{q-1}{q} \sum_{n} F_{n}^{*}(t) \widehat{P}_{n} .
$$


Учитывая, что

$$
\left[1-P_{\text {rel }}(t)\right] i L_{N} \varrho_{\text {rel }}(t)=-\sum_{n} I_{n}(t) F_{n}(t) \varrho_{\text {rel }}(t)
$$

с обобщенными потоками

$$
I_{n}(t)=[1-P(t)] \frac{1}{q} \psi^{-1}(t) \dot{\widehat{P}}_{n}
$$

мы можем записать неравновесный статистический оператор в явном виде:

$$
\varrho\left(x^{N} ; t\right)=\varrho_{\text {rel }}\left(x^{N} ; t\right)-\sum_{n} \int_{-\infty}^{t} e^{\varepsilon\left(t^{\prime}-t\right)} T\left(t, t^{\prime}\right) I_{n}\left(t^{\prime}\right) F_{n}\left(t^{\prime}\right) \varrho_{\text {rel }}\left(x^{N} ; t^{\prime}\right) d t^{\prime} .
$$

С его помощью можно получить обобщенные уравнения переноса для параметров сокращенного описания, которые мы представим следующим образом:

$$
\frac{\partial}{\partial t}\left\langle\widehat{P}_{m}\right\rangle^{t}=\left\langle\dot{\widehat{P}}_{m}\right\rangle_{\text {rel }}^{t}+\sum_{n} \int_{-\infty}^{t} e^{\varepsilon\left(t^{\prime}-t\right)} \varphi_{m n}\left(t, t^{\prime}\right) F_{n}\left(t^{\prime}\right) d t^{\prime} .
$$

Первый член в правой части уравнения (16) рассчитывается с помощью релевантного статистического оператора (10). Обобщенные ядра переноса (функции памяти)

$$
\varphi_{m n}\left(t, t^{\prime}\right)=\int \dot{\widehat{P}}_{m} T\left(t, t^{\prime}\right) I_{n}\left(t^{\prime}\right) \varrho_{\mathrm{rel}}\left(t^{\prime}\right) d \Gamma_{N}
$$

представляют собой временны́е корреляционные функции (с учетом редуцированного оператора эволюции (2)), которые описывают диссипативные процессы в системе и построены на обобщенных потоках $I_{n}(t)$. Уравнения переноса $(16)$, в общем случае учитывающие эффекты памяти, в приближении $\varphi_{m n}\left(t, t^{\prime}\right) \approx \varphi_{m n} \delta\left(t-t^{\prime}\right)$ описывают марковские процессы. Уравнения переноса являются незамкнутыми. Неравновесные множители Лагранжа в них (неравновесные термодинамические параметры в случае гидродинамического описания) определяются из условий самосогласования (9). С этой точки зрения система уравнений переноса становится замкнутой. Неравновесный статистический оператор (15) и уравнения переноса (16) составляют полный набор инструментов для описания неравновесных процессов в системах, далеких от равновесия, когда определены параметры сокращенного описания $\left\langle\widehat{P}_{n}\right\rangle^{t}$.

В следующем разделе мы применим представленный подход к описанию нелинейных кинетических флуктуаций в газах и жидкостях, далеких от равновесия.

\section{3. ОБОБЩЕННЫЕ КИНЕТИЧЕСКИЕ УРАВНЕНИЯ В СТАТИСТИКЕ РЕНЬИ}

Для описания кинетических процессов в классических газах и жидкостях, далеких от равновесия, в качестве параметров сокращенного описания могут быть выбраны неравновесные одно- и двухчастичная функции распределения

$$
f_{1}(x ; t)=\left\langle\hat{n}_{1}(x)\right\rangle^{t}, \quad f_{2}\left(x, x^{\prime} ; t\right)=\left\langle\hat{n}_{2}\left(x, x^{\prime}\right)\right\rangle^{t},
$$

где

$$
\hat{n}_{1}(x)=\sum_{j=1}^{N} \delta\left(x-x_{j}\right), \quad \hat{n}_{2}\left(x, x^{\prime}\right)=\sum_{j=1}^{N} \sum_{l=1}^{N} \delta\left(x-x_{j}\right) \delta\left(x^{\prime}-x_{l}\right)
$$


- одно- и двухчастичная микроскопические фазовые плотности $N$ частиц в объеме $V$. Они определяют плотности числа частиц, импульса и полной энергии (представленной суммой кинетической $\hat{\varepsilon}^{\mathrm{kin}}$ и потенциальной $\hat{\varepsilon}^{\mathrm{int}}$ частей)

$$
\begin{aligned}
\hat{n}(\vec{r}) & =\int \hat{n}_{1}(\vec{r}, \vec{p}) d \vec{p}, \quad \hat{\vec{p}}(\vec{r})=\int \hat{n}_{1}(\vec{r}, \vec{p}) \vec{p} d \vec{p}, \\
\hat{\varepsilon}^{\operatorname{kin}}(\vec{r}) & =\int \hat{n}_{1}(\vec{r}, \vec{p}) \frac{p^{2}}{2 m} d \vec{p}, \\
\hat{\varepsilon}^{\operatorname{int}}(\vec{r}) & =\frac{1}{2} \int d \vec{p} \int d \vec{p}^{\prime} \int \Phi\left(\left|\vec{r}-\vec{r}^{\prime}\right|\right) \hat{n}_{2}\left(\vec{r}, \vec{p} ; \vec{r}^{\prime}, \vec{p}^{\prime}\right) d \vec{r}^{\prime},
\end{aligned}
$$

средние значения которых

$$
\begin{aligned}
\langle\hat{n}(\vec{r})\rangle^{t} & =\int f_{1}(\vec{r}, \vec{p} ; t) d \vec{p}, \quad\langle\hat{\vec{p}}(\vec{r})\rangle^{t}=\int f_{1}(\vec{r}, \vec{p} ; t) \vec{p} d \vec{p}, \\
\left\langle\hat{\varepsilon}^{\operatorname{kin}}(\vec{r})\right\rangle^{t} & =\int f_{1}(\vec{r}, \vec{p} ; t) \frac{p^{2}}{2 m} d \vec{p}, \\
\left\langle\hat{\varepsilon}^{\operatorname{int}}(\vec{r})\right\rangle^{t} & =\frac{1}{2} \int d \vec{p} \int d \vec{p}^{\prime} \int \Phi\left(\left|\vec{r}-\vec{r}^{\prime}\right|\right) f_{2}\left(\vec{r}, \vec{p} ; \vec{r}^{\prime}, \vec{p}^{\prime} ; t\right) d \vec{r}^{\prime}
\end{aligned}
$$

удовлетворяют локальным законам сохранения:

$$
\begin{aligned}
& \frac{\partial}{\partial t}\langle\hat{n}(\vec{r})\rangle^{t}=-\frac{1}{m} \vec{\nabla} \cdot\langle\hat{\vec{p}}(\vec{r})\rangle^{t}, \\
& \frac{\partial}{\partial t}\langle\hat{\vec{p}}(\vec{r})\rangle^{t}=-\vec{\nabla} \cdot\left(\left\langle\widehat{\leftrightarrow}^{\mathrm{kin}}(\vec{r})\right\rangle^{t}+\left\langle\widehat{\leftrightarrow}^{\mathrm{int}}(\vec{r})\right\rangle^{t}\right), \\
& \frac{\partial}{\partial t}\langle\hat{\varepsilon}(\vec{r})\rangle^{t}=-\vec{\nabla} \cdot\left(\left\langle\hat{\vec{j}}_{E}^{\operatorname{kin}}(\vec{r})\right\rangle^{t}+\left\langle\hat{\vec{j}}_{E}^{\mathrm{int}}(\vec{r})\right\rangle^{t}\right) .
\end{aligned}
$$

Здесь $\langle\hat{\varepsilon}(\vec{r})\rangle^{t}=\left\langle\hat{\varepsilon}^{\operatorname{kin}}(\vec{r})\right\rangle^{t}+\left\langle\hat{\varepsilon}^{\mathrm{int}}(\vec{r})\right\rangle^{t}-$ неравновесное среднее значение плотности полной энергии, $\vec{\nabla}=\partial / \partial \vec{r}$,

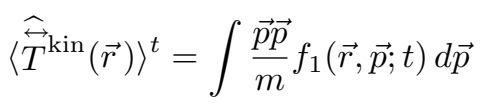

- неравновесное среднее значение плотности кинетической части тензора напряжений,

$$
\begin{aligned}
& \left\langle\widehat{\stackrel{T}{T}}^{\text {int }}(\vec{r})\right\rangle^{t}= \\
& \quad=\frac{1}{2} \int d \vec{p} \int d \vec{p}^{\prime} \int \frac{\partial}{\partial\left|\vec{r}-\vec{r}^{\prime}\right|} \Phi\left(\left|\vec{r}-\vec{r}^{\prime}\right|\right) \frac{\left(\vec{r}-\vec{r}^{\prime}\right)\left(\vec{r}-\vec{r}^{\prime}\right)}{\left|\vec{r}-\vec{r}^{\prime}\right|} f_{2}\left(\vec{r}, \vec{r}^{\prime} ; \vec{p}, \vec{p}^{\prime} ; t\right) d \vec{r}^{\prime}
\end{aligned}
$$

- неравновесное среднее значение плотности потенциальной части тензора напряжений,

$$
\left\langle\hat{\vec{j}}_{E}^{\mathrm{kin}}(\vec{r})\right\rangle^{t}=\int \frac{p^{2}}{2 m} \vec{p} f_{1}(\vec{r}, \vec{p} ; t) d \vec{p}
$$

и

$$
\left\langle\hat{\vec{j}}_{E}^{\mathrm{int}}(\vec{r})\right\rangle^{t}=\int d \vec{p} \int d \vec{p}^{\prime} \int\left[\frac{\vec{p}}{m} \Phi\left(\left|\vec{r}-\vec{r}^{\prime}\right|\right)-\Phi\left(\left|\vec{r}-\vec{r}^{\prime}\right|\right) \frac{\vec{r}-\vec{r}^{\prime}}{\left|\vec{r}-\vec{r}^{\prime}\right|}\right] f_{2}\left(\vec{r}, \vec{r}^{\prime} ; \vec{p}, \vec{p}^{\prime} ; t\right) d \vec{r}^{\prime}
$$


- неравновесные средние значения плотности кинетической и потенциальной частей потока энергии соответственно. Из приведенных соотношений следует, что неравновесная одночастичная функция распределения определяет макроскопические неравновесные плотности числа частиц, импульса, а также кинетические части полной энергии, тензора напряжений и потока энергии, тогда как неравновесная двухчастичная функция распределения определяет потенциальные части полной энергии, тензора напряжений и потока энергии. Таким образом, в системах, далеких от равновесия, нелинейные гидродинамические флуктуации обусловлены нелинейными флуктуациями неравновесных одно- и двухчастичной функций распределения, для которых следует построить соответствующие кинетические уравнения. В таком случае, когда неравновесные одно- и двухчастичная функции распределения $f_{1}(x ; t)=\left\langle\hat{n}_{1}(x)\right\rangle^{t}$ и $f_{2}\left(x, x^{\prime} ; t\right)=\left\langle\hat{n}_{2}\left(x, x^{\prime}\right)\right\rangle^{t}$ выбраны в качестве параметров сокращенного описания, согласно (7) релевантная функция распределения имеет вид

$$
\begin{aligned}
\varrho_{\mathrm{rel}}(t)= & \frac{1}{Z_{\mathrm{R}}(t)}\left\{1-\frac{q-1}{q}\left[\int x a(x ; t) \delta \hat{n}_{1}(x ; t) d x+\right.\right. \\
& \left.\left.+\int d x \int b\left(x, x^{\prime} ; t\right) \delta \hat{n}_{2}\left(x, x^{\prime} ; t\right) d \vec{x}^{\prime}\right]\right\}^{1 /(q-1)},
\end{aligned}
$$

где

$$
\begin{aligned}
Z_{\mathrm{R}}(t)=\int & d \Gamma_{N}\left\{1-\frac{q-1}{q}\left[\int a(x ; t) \delta \hat{n}_{1}(x ; t) d x+\right.\right. \\
& \left.\left.+\int d x \int b\left(x, x^{\prime} ; t\right) \delta \hat{n}_{2}\left(x, x^{\prime} ; t\right) d x^{\prime}\right]\right\}^{1 /(q-1)}
\end{aligned}
$$

- статистическая сумма релевантной функции распределения. Параметры $a(x ; t)$ и $b\left(x, x^{\prime} ; t\right)$ определяются из следующих условий самосогласования:

$$
\left\langle\hat{n}_{1}(x)\right\rangle^{t}=\left\langle\hat{n}_{1}(x)\right\rangle_{\mathrm{rel}}^{t}, \quad\left\langle\hat{n}_{2}\left(x, x^{\prime}\right)\right\rangle^{t}=\left\langle\hat{n}_{2}\left(x, x^{\prime}\right)\right\rangle_{\mathrm{rel}}^{t}
$$

Релевантную функцию распределения (20) удобно представить в другой форме:

$$
\begin{aligned}
\varrho_{\mathrm{rel}}(t)= & \frac{1}{Z_{\mathrm{R}}^{*}(t)}\left\{1-\frac{q-1}{q}\left[\int a^{*}(x ; t) \hat{n}_{1}(x) d x+\right.\right. \\
& \left.\left.\quad+\int d x \int b^{*}\left(x, x^{\prime} ; t\right) \hat{n}_{2}\left(x, x^{\prime}\right) d x^{\prime}\right]\right\}^{1 /(q-1)},
\end{aligned}
$$

записав множители Лагранжа следующим образом:

$$
\begin{aligned}
a^{*}(x ; t)=a & (x ; t)\left\{1+\frac{q-1}{q} \times\right. \\
& \left.\times\left[\int a(x ; t) f_{1}(x ; t) d x+\int d x \int b\left(x, x^{\prime} ; t\right) f_{2}\left(x, x^{\prime} ; t\right) d x^{\prime}\right]\right\}^{-1}, \\
b^{*}\left(x, x^{\prime} ; t\right)= & b\left(x, x^{\prime} ; t\right)\left\{1+\frac{q-1}{q} \times\right. \\
\times & {\left.\left[\int a(x ; t) f_{1}(x ; t) d x+\int d x \int b\left(x, x^{\prime} ; t\right) f_{2}\left(x, x^{\prime} ; t\right) d x^{\prime}\right]\right\}^{-1} . }
\end{aligned}
$$


Важно отметить, что в случае $q=1$ имеем $a^{*}(x ; t)=a(x ; t)$ и $b^{*}\left(x, x^{\prime} ; t\right)=$ $b\left(x, x^{\prime} ; t\right)$, и мы получим релевантную функцию распределения, соответствующую статистике Гиббса [24].

Теперь мы можем представить неравновесный статистический оператор системы следующим образом:

$$
\begin{aligned}
& \varrho(t)=\varrho_{\text {rel }}(t)+\int d x^{\prime} \int_{-\infty}^{t} e^{\varepsilon\left(t^{\prime}-t\right)} T\left(t, t^{\prime}\right) a\left(x^{\prime} ; t^{\prime}\right) I_{n}^{(1)}\left(x^{\prime} ; t^{\prime}\right) \varrho_{\text {rel }}(t) d t^{\prime}+ \\
& \quad+\int d x^{\prime} \int d x^{\prime \prime} \int_{-\infty}^{t} e^{\varepsilon\left(t^{\prime}-t\right)} T\left(t, t^{\prime}\right) b\left(x^{\prime}, x^{\prime \prime} ; t^{\prime}\right) I_{n}^{(2)}\left(x^{\prime}, x^{\prime \prime} ; t^{\prime}\right) \varrho_{\text {rel }}(t) d t^{\prime},
\end{aligned}
$$

где

$$
\begin{aligned}
I_{n}^{(1)}(x ; t) & =[1-P(t)] \frac{1}{q} \psi^{-1}(t) i L_{N} \hat{n}_{1}(x), \\
I_{n}^{(2)}\left(x, x^{\prime} ; t\right) & =[1-P(t)] \frac{1}{q} \psi^{-1}(t) i L_{N} \hat{n}_{2}\left(x, x^{\prime}\right)
\end{aligned}
$$

- обобщенные потоки, в которых функция $\psi(t)$ равна

$$
\psi(t)=1-\frac{q-1}{q}\left[\int a^{*}(x ; t) \hat{n}_{1}(x ; t) d x+\int d x \int b^{*}\left(x, x^{\prime} ; t\right) \hat{n}_{2}\left(x, x^{\prime} ; t\right) d x^{\prime}\right] .
$$

С помощью неравновесного статистического оператора (23) согласно (16) мы получим систему обобщенных кинетических уравнений для параметров сокращенного описания $f_{1}(x ; t)=\left\langle\hat{n}_{1}(x)\right\rangle^{t}$ и $f_{2}\left(x, x^{\prime} ; t\right)=\left\langle\hat{n}_{2}\left(x, x^{\prime}\right)\right\rangle^{t}$ :

$$
\begin{aligned}
\frac{\partial}{\partial t}\left\langle\hat{n}_{1}(x)\right\rangle^{t}=\int & \Phi_{n n}^{11}\left(x, x^{\prime} ; t\right) a\left(x^{\prime} ; t\right) d x^{\prime}+ \\
& +\int d x^{\prime} \int \Phi_{n n}^{12}\left(x ; x^{\prime}, x^{\prime \prime} ; t\right) b\left(x^{\prime}, x^{\prime \prime} ; t\right) d x^{\prime \prime}+ \\
& +\int d x^{\prime} \int_{-\infty}^{t} e^{\varepsilon\left(t^{\prime}-t\right)} \varphi_{n n}^{11}\left(x, x^{\prime} ; t, t^{\prime}\right) a\left(x^{\prime} ; t^{\prime}\right) d t^{\prime}+ \\
& +\int d x^{\prime} \int d x^{\prime \prime} \int_{-\infty}^{t} e^{\varepsilon\left(t^{\prime}-t\right)} \varphi_{n n}^{12}\left(x ; x^{\prime}, x^{\prime \prime} ; t, t^{\prime}\right) b\left(x^{\prime}, x^{\prime \prime} ; t^{\prime}\right) d t^{\prime}, \\
\frac{\partial}{\partial t}\left\langle\hat{n}_{2}\left(x, x^{\prime}\right)\right\rangle^{t}=\int & \Phi_{n n}^{21}\left(x, x^{\prime} ; x^{\prime \prime} ; t\right) a\left(x^{\prime \prime} ; t\right) d x^{\prime \prime}+ \\
& +\int d x^{\prime \prime} \int \Phi_{n n}^{22}\left(x ; x^{\prime} ; x^{\prime \prime}, x^{\prime \prime \prime} ; t\right) b\left(x^{\prime \prime}, x^{\prime \prime \prime} ; t\right) d x^{\prime \prime \prime}+ \\
& +\int d x^{\prime \prime} \int_{-\infty}^{t} e^{\varepsilon\left(t^{\prime}-t\right)} \varphi_{n n}^{21}\left(x, x^{\prime} ; x^{\prime \prime} t, t^{\prime}\right) a\left(x^{\prime \prime} ; t^{\prime}\right) d t^{\prime}+ \\
& +\int d x^{\prime \prime} \int^{t} d x^{\prime \prime \prime} \int_{-\infty}^{t} e^{\varepsilon\left(t^{\prime}-t\right)} \varphi_{n n}^{22}\left(x ; x^{\prime} ; x^{\prime \prime}, x^{\prime \prime \prime} ; t, t^{\prime}\right) b\left(x^{\prime \prime}, x^{\prime \prime \prime} ; t^{\prime}\right) d t^{\prime} .
\end{aligned}
$$

В этих уравнениях функции

$$
\Phi_{P P}^{\alpha \beta}\left(x, x^{\prime} ; t\right)=\int \widehat{P}_{\alpha}(x) \frac{1}{q} \psi^{-1} i L_{N} \widehat{P}_{\beta}\left(x^{\prime}\right) \varrho_{\mathrm{rel}}\left(x^{N} ; t\right) d \Gamma_{N}
$$


описывают недиссипативную динамику, а функции

$$
\varphi_{I_{P} I_{P}}^{\alpha \beta}\left(x ; x^{\prime} ; t, t^{\prime}\right)=\int i L_{N} \widehat{P}_{\alpha}(x) T\left(t, t^{\prime}\right) I_{P}^{\beta}\left(x^{\prime} ; t^{\prime}\right) \varrho_{\text {rel }}\left(x^{N} ; t^{\prime}\right) d \Gamma_{N}
$$

- кинетические ядра переноса, которые описывают диссипативные процессы. Здесь мы ввели обозначение $\widehat{P}_{\alpha}(x)=\left\{\hat{n}_{1}(x), \hat{n}_{2}\left(x, x^{\prime}\right)\right\}$. Если пренебречь двухчастичными корреляциями при $q=1$, обобщенное кинетическое уравнение в статистике Реньи переходит в кинетическое уравнение, полученное в рамках статистики Гиббса [30], в котором ядра переноса рассчитываются при помощи релевантной функции распределения $\varrho_{\text {rel }}(t)=\prod_{j=1}^{N}\left[f_{1}\left(x_{j} ; t\right) / e\right]$. В этом случае неравновесный статистический оператор следует искать как решение уравнения Лиувилля с граничным условием, соответствующим гипотезе Боголюбова об ослаблении корреляций между частицами:

$$
\frac{\partial}{\partial t} \varrho\left(x^{N} ; t\right)+i L_{N} \varrho\left(x^{N} ; t\right)=-\varepsilon\left(\varrho\left(x^{N} ; t\right)-\prod_{j=1}^{N} \frac{f_{1}\left(x_{j} ; t\right)}{e}\right) .
$$

Для более детального изучения структуры корреляционных функций (25) и ядер переноса (26) рассмотрим действие оператора Лиувилля на $\hat{n}_{1}(x)$ и $\hat{n}_{2}\left(x, x^{\prime}\right)$. Его можно записать следующим образом:

$$
i L_{N} \hat{n}_{1}(x)=-\frac{\partial}{\partial \vec{r}} \cdot \frac{1}{m} \hat{\vec{j}}(\vec{r}, \vec{p})+\frac{\partial}{\partial \vec{p}} \cdot \hat{\vec{F}}(\vec{r}, \vec{p}),
$$

где

$$
\hat{\vec{j}}(\vec{r}, \vec{p})=\sum_{j=1}^{N} \vec{p}_{j} \delta\left(\vec{r}-\vec{r}_{j}\right) \delta\left(\vec{p}-\vec{p}_{j}\right)
$$

- микроскопическая плотность импульса в пространстве координат и импульсов, а

$$
\hat{\vec{F}}(\vec{r}, \vec{p})=\sum_{l \neq j} \frac{\partial}{\partial \vec{r}_{j}} \Phi\left(\left|\vec{r}_{j}-\vec{r}_{l}\right|\right) \delta\left(\vec{r}-\vec{r}_{j}\right) \delta\left(\vec{p}-\vec{p}_{j}\right)
$$

- микроскопическая плотность силы в пространстве координат и импульсов. Аналогично имеем

$$
\begin{aligned}
i L_{N} \hat{n}_{2}\left(x, x^{\prime}\right)=- & \frac{\partial}{\partial \vec{r}} \cdot \frac{1}{m} \hat{\vec{j}}(\vec{r}, \vec{p}) \hat{n}_{1}\left(x^{\prime}\right)-\hat{n}_{1}(x) \frac{\partial}{\partial \vec{r}^{\prime}} \cdot \frac{1}{m} \hat{\vec{j}}\left(\vec{r}^{\prime}, \vec{p}^{\prime}\right)+ \\
& +\frac{\partial}{\partial \vec{p}} \cdot \hat{\vec{F}}(\vec{r}, \vec{p}) \hat{n}_{1}\left(x^{\prime}\right)+\hat{n}_{1}(x) \frac{\partial}{\partial \vec{p}^{\prime}} \cdot \hat{\vec{F}}\left(\vec{r}^{\prime}, \vec{p}^{\prime}\right) .
\end{aligned}
$$

Принимая во внимание соотношения (27)-(30), для функций (25) мы получим, в частности,

$$
\Phi_{n n}^{11}\left(x, x^{\prime} ; t\right)=\left[\Omega_{n j}\left(x, x^{\prime} ; t\right) \cdot \frac{\partial}{\partial \vec{r}^{\prime}}-\Omega_{n F}\left(x, x^{\prime} ; t\right) \cdot \frac{\partial}{\partial \vec{p}^{\prime}}\right] .
$$


Обобщенные ядра переноса (26) можно представить следующим образом:

$$
\begin{aligned}
& \varphi_{n n}^{11}\left(x, x^{\prime} ; t, t^{\prime}\right)=-\left[\frac{\partial}{\partial \vec{r}} \cdot D_{j j}\left(x, x^{\prime} ; t, t^{\prime}\right) \cdot \frac{\partial}{\partial \vec{r}^{\prime}}-\frac{\partial}{\partial \vec{p}} \cdot D_{F j}\left(x, x^{\prime} ; t, t^{\prime}\right) \cdot \frac{\partial}{\partial \vec{r}^{\prime}}-\right. \\
& \left.-\frac{\partial}{\partial \vec{r}} \cdot D_{j F}\left(x, x^{\prime} ; t, t^{\prime}\right) \cdot \frac{\partial}{\partial \vec{p}^{\prime}}+\frac{\partial}{\partial \vec{p}} \cdot D_{F F}\left(x, x^{\prime} ; t, t^{\prime}\right) \cdot \frac{\partial}{\partial \vec{p}^{\prime}}\right], \\
& \varphi_{n n}^{12}\left(x, x^{\prime}, x^{\prime \prime} ; t, t^{\prime}\right)= \\
& =-\left[\frac{\partial}{\partial \vec{r}} \cdot D_{j j n}\left(x, x^{\prime}, x^{\prime \prime} ; t, t^{\prime}\right) \cdot \frac{\partial}{\partial \vec{r}^{\prime}}+\frac{\partial}{\partial \vec{r}} \cdot D_{j n j}\left(x, x^{\prime}, x^{\prime \prime} ; t, t^{\prime}\right) \cdot \frac{\partial}{\partial \vec{r}^{\prime \prime}}-\right. \\
& -\frac{\partial}{\partial \vec{p}} \cdot D_{F j n}\left(x, x^{\prime}, x^{\prime \prime} ; t, t^{\prime}\right) \cdot \frac{\partial}{\partial \vec{r}^{\prime}}-\frac{\partial}{\partial \vec{p}} \cdot D_{F n j}\left(x, x^{\prime}, x^{\prime \prime} ; t, t^{\prime}\right) \cdot \frac{\partial}{\partial \vec{r}^{\prime \prime}}- \\
& -\frac{\partial}{\partial \vec{r}} \cdot D_{j F n}\left(x, x^{\prime}, x^{\prime \prime} ; t, t^{\prime}\right) \cdot \frac{\partial}{\partial \vec{p}^{\prime}}-\frac{\partial}{\partial \vec{r}} \cdot D_{j n F}\left(x, x^{\prime}, x^{\prime \prime} ; t, t^{\prime}\right) \cdot \frac{\partial}{\partial \vec{p}^{\prime \prime}}+ \\
& \left.+\frac{\partial}{\partial \vec{p}} \cdot D_{F F n}\left(x, x^{\prime}, x^{\prime \prime} ; t, t^{\prime}\right) \cdot \frac{\partial}{\partial \vec{p}^{\prime}}+\frac{\partial}{\partial \vec{p}} \cdot D_{F n F}\left(x, x^{\prime}, x^{\prime \prime} ; t, t^{\prime}\right) \cdot \frac{\partial}{\partial \vec{p}^{\prime \prime}}\right], \\
& \varphi_{n n}^{22}\left(x, x^{\prime}, x^{\prime \prime}, x^{\prime \prime \prime} ; t, t^{\prime}\right)= \\
& =-\frac{\partial}{\partial \vec{r}} \cdot\left[D_{j n j n}\left(x, x^{\prime}, x^{\prime \prime}, x^{\prime \prime \prime} ; t, t^{\prime}\right) \cdot \frac{\partial}{\partial \vec{r}^{\prime \prime}}+D_{j n n j}\left(x, x^{\prime}, x^{\prime \prime}, x^{\prime \prime \prime} ; t, t^{\prime}\right) \cdot \frac{\partial}{\partial \vec{r}^{\prime \prime \prime}}\right]- \\
& -\frac{\partial}{\partial \vec{r}^{\prime}} \cdot\left[D_{n j j n}\left(x, x^{\prime}, x^{\prime \prime}, x^{\prime \prime \prime} ; t, t^{\prime}\right) \cdot \frac{\partial}{\partial \vec{r}^{\prime \prime}}+D_{n j n j}\left(x, x^{\prime}, x^{\prime \prime}, x^{\prime \prime \prime} ; t, t^{\prime}\right) \cdot \frac{\partial}{\partial \vec{r}^{\prime \prime \prime}}\right]+ \\
& +\frac{\partial}{\partial \vec{p}} \cdot\left[D_{F n j n}\left(x, x^{\prime}, x^{\prime \prime}, x^{\prime \prime \prime} ; t, t^{\prime}\right) \cdot \frac{\partial}{\partial \vec{r}^{\prime \prime}}+D_{F n n j}\left(x, x^{\prime}, x^{\prime \prime}, x^{\prime \prime \prime} ; t, t^{\prime}\right) \cdot \frac{\partial}{\partial \vec{r}^{\prime \prime \prime}}\right]+ \\
& +\frac{\partial}{\partial \vec{p}^{\prime}} \cdot\left[D_{n F j n}\left(x, x^{\prime}, x^{\prime \prime}, x^{\prime \prime \prime} ; t, t^{\prime}\right) \cdot \frac{\partial}{\partial \vec{r}^{\prime \prime}}+D_{n F n j}\left(x, x^{\prime}, x^{\prime \prime}, x^{\prime \prime \prime} ; t, t^{\prime}\right) \cdot \frac{\partial}{\partial \vec{r}^{\prime \prime \prime}}\right]+ \\
& +\frac{\partial}{\partial \vec{r}} \cdot\left[D_{j n F n}\left(x, x^{\prime}, x^{\prime \prime}, x^{\prime \prime \prime} ; t, t^{\prime}\right) \cdot \frac{\partial}{\partial \vec{p}^{\prime \prime}}+D_{j n n F}\left(x, x^{\prime}, x^{\prime \prime}, x^{\prime \prime \prime} ; t, t^{\prime}\right) \cdot \frac{\partial}{\partial \vec{p}^{\prime \prime \prime}}\right]+ \\
& +\frac{\partial}{\partial \vec{r}^{\prime}} \cdot\left[D_{n j F n}\left(x, x^{\prime}, x^{\prime \prime}, x^{\prime \prime \prime} ; t, t^{\prime}\right) \cdot \frac{\partial}{\partial \vec{p}^{\prime \prime}}+D_{n j n F}\left(x, x^{\prime}, x^{\prime \prime}, x^{\prime \prime \prime} ; t, t^{\prime}\right) \cdot \frac{\partial}{\partial \vec{p}^{\prime \prime \prime}}\right]- \\
& -\frac{\partial}{\partial \vec{p}} \cdot\left[D_{F n F n}\left(x, x^{\prime}, x^{\prime \prime}, x^{\prime \prime} ; t, t^{\prime}\right) \cdot \frac{\partial}{\partial \vec{p}^{\prime \prime}}+D_{F n n F}\left(x, x^{\prime}, x^{\prime \prime}, x^{\prime \prime \prime} ; t, t^{\prime}\right) \cdot \frac{\partial}{\partial \vec{p}^{\prime \prime \prime}}\right]- \\
& -\frac{\partial}{\partial \vec{p}^{\prime}} \cdot\left[D_{n F F n}\left(x, x^{\prime}, x^{\prime \prime}, x^{\prime \prime} ; t, t^{\prime}\right) \cdot \frac{\partial}{\partial \vec{p}^{\prime \prime}}+D_{n F n F}\left(x, x^{\prime}, x^{\prime \prime}, x^{\prime \prime \prime} ; t, t^{\prime}\right) \cdot \frac{\partial}{\partial \vec{p}^{\prime \prime \prime}}\right] \text {, }
\end{aligned}
$$

где

$$
\begin{aligned}
D_{j j}\left(x, x^{\prime} ; t, t^{\prime}\right) & =\int \hat{\vec{j}}(x) T\left(t, t^{\prime}\right)\left[1-P\left(t^{\prime}\right)\right] \frac{1}{q} \psi^{-1}(t) \hat{\vec{j}}\left(x^{\prime}\right) \varrho_{\mathrm{rel}}\left(x^{N} ; t^{\prime}\right) d \Gamma_{N}, \\
D_{F F}\left(x, x^{\prime} ; t, t^{\prime}\right) & =\int \hat{\vec{F}}(x) T\left(t, t^{\prime}\right)\left[1-P\left(t^{\prime}\right)\right] \frac{1}{q} \psi^{-1}(t) \hat{\vec{F}}\left(x^{\prime}\right) \varrho_{\mathrm{rel}}\left(x^{N} ; t^{\prime}\right) d \Gamma_{N}
\end{aligned}
$$

- обобщенные коэффициенты диффузии и трения в пространстве координат и импульсов в рамках статистики Реньи. При этом в случае $q=1$ они переходят в обоб- 
щенные коэффициенты диффузии и трения в статистике Гиббса

$$
\begin{aligned}
& \int d \vec{p} \int D_{j j}\left(x, x^{\prime} ; t, t^{\prime}\right) d \vec{p}^{\prime}=D_{j j}\left(\vec{r}, \vec{r}^{\prime} ; t, t^{\prime}\right), \\
& \int d \vec{p} \int D_{F F}\left(x, x^{\prime} ; t, t^{\prime}\right) d \vec{p}^{\prime}=D_{F F}\left(\vec{r}, \vec{r}^{\prime} ; t, t^{\prime}\right) .
\end{aligned}
$$

Полученные кинетические уравнения содержат корреляционные функции второго, третьего и четвертого порядков $\Omega_{n j}, \Omega_{n F}, \Omega_{n n j}, \Omega_{n n F}, \Omega_{n n j n}, \Omega_{n n F n}$ по динамическим переменным $\hat{n}(x), \widehat{\vec{j}}(x), \hat{\vec{F}}(x)$. Величины $\Omega_{\alpha \beta}$ представляют собой корреляционные функции, описывающие недиссипативные процессы. Обобщенные функции памяти $D_{\alpha \beta}, D_{\alpha \beta \gamma}$ и $D_{\alpha \beta \gamma \delta}$ представляют собой временнь́е корреляционные функции, построенные на динамических переменных $\hat{n}(x), \hat{\vec{j}}(x), \hat{\vec{F}}(x),[1-P(t)] \hat{\vec{j}}(x)$, $[1-P(t)] \hat{\vec{F}}(x)$, которые описывают немарковские диссипативные процессы в системе. При $q=1$ они переходят в функции памяти в статистике Гиббса. Такие функции памяти как $D_{n j n j}$ и $D_{n F n F}$ имеют сложную структуру:

$$
\begin{aligned}
& D_{n j n j}\left(x, x^{\prime}, x^{\prime \prime}, x^{\prime \prime \prime} ; t, t^{\prime}\right)= \\
& \quad=\int \hat{n}(x) \hat{\vec{j}}\left(x^{\prime}\right) T\left(t, t^{\prime}\right)\left[1-P\left(t^{\prime}\right)\right] \frac{1}{q} \psi^{-1}(t) \hat{n}\left(x^{\prime \prime}\right) \hat{\vec{j}}\left(x^{\prime \prime \prime}\right) \varrho_{\mathrm{rel}}\left(x^{N} ; t^{\prime}\right) d \Gamma_{N}, \\
& D_{n F n F}\left(x, x^{\prime}, x^{\prime \prime}, x^{\prime \prime \prime} ; t, t^{\prime}\right)= \\
& \quad=\int \hat{n}(x) \hat{\vec{F}}\left(x^{\prime}\right) T\left(t, t^{\prime}\right)\left[1-P\left(t^{\prime}\right)\right] \frac{1}{q} \psi^{-1}(t) \hat{n}\left(x^{\prime \prime}\right) \hat{\vec{F}}\left(x^{\prime \prime \prime}\right) \varrho_{\mathrm{rel}}\left(x^{N} ; t^{\prime}\right) d \Gamma_{N},
\end{aligned}
$$

тем не менее их можно аппроксимировать, например, следующим образом:

$$
D_{n j n j} \approx D_{n n} D_{j j}+D_{n j} D_{j n}, \quad D_{n F n F} \approx D_{n n} D_{F F}+D_{n F} D_{F n},
$$

что отвечает идеологии теории взаимодействующих мод.

Обобщенные кинетические уравнения (24) с учетом (31), (32) имеют структуру типа уравнения Фоккера-Планка. Их можно использовать для перехода к уравнениям обобщенной гидродинамики для локально сохраняющихся величин (19): плотностей числа частиц, импульса и энергии. Действительно, умножая систему уравнений (24) на первые моменты неравновесной одночастичной функции распределения $f_{1}(\vec{r}, \vec{p} ; t)\left(1, \vec{p}, p^{2} / 2 m\right)$ и на $\frac{1}{2} \Phi\left(\left|\vec{r}-\vec{r}^{\prime}\right|\right)$, мы получим обобщенные уравнения гидродинамики, в которых коэффициенты обобщенной вязкости и теплопроводимости определяются через ядра переноса с разделенными вкладами от кинетической и потенциальной частей энергии.

В случае разреженных газов в состояниях, близких к равновесию, когда можно пренебречь неравновесными парными корреляциями, описывающимися функцией $\left\langle\hat{n}_{2}\left(x, x^{\prime}\right)\right\rangle^{t}$, можно воспроизвести результаты, подобные результатам Богосяна [72].

Актуальной является переформулировка представленного подхода для описания диссипативных процессов в открытых системах [73]-[75], диссипации и диффузии в реакциях ядерного распада и синтеза [75] и квантовой турбулентности [1], [76]. Для описания диссипативных процессов в открытых квантовых системах широко применяется уравнение Линдблада [77]-[80]. Оно может быть получено с помощью q-обобщения уравнения Лиувилля [67], которое мы рассмотрим в следующем разделе. 


\section{4. q-ОБОБЩЕНИЕ УРАВНЕНИЯ ЛИУВИЛЛЯ}

Интересное $q$-обобщение обычного уравнения Лиувилля было представлено в работе [67]:

$$
\frac{\partial}{\partial t} \varrho\left(x^{N} ; t\right)+i \widetilde{L}_{N}(t) \varrho\left(x^{N} ; t\right)=0,
$$

где $i \widetilde{L}_{N}-q$-параметризированный оператор Лиувилля

$$
i \widetilde{L}_{N}(t)=\frac{i L_{N}}{1+(1-q) t i L_{N}},
$$

который при $q=1$ переходит в оператор Лиувилля (3). Когда $|1-q| \Omega_{\mathrm{ch}} t \ll 1$, где $\Omega_{\mathrm{ch}}$ - характеристическая частота рассматриваемой физической системы, уравнение (33) можно записать в следующем виде [67]:

$$
\frac{\partial}{\partial t} \varrho\left(x^{N} ; t\right)+\left[i L_{N}-(1-q) t\left(i L_{N}\right)^{2}\right] \varrho\left(x^{N} ; t\right)=0 .
$$

Это уравнение типа Линдблада для неравновесного статистического оператора $\varrho\left(x^{N} ; t\right)$. Уравнение типа Линдблада в рамках статистики Реньи было получено в работе [48]. Решение $q$-параметризированного уравнения Лиувилля в рамках метода неравновесного статистического оператора можно представить следующим образом:

$$
\begin{aligned}
\varrho\left(x^{N} ; t\right)= & -\varepsilon \int_{-\infty}^{t} e^{\varepsilon\left(t^{\prime}-t\right)} T_{q}\left(t, t^{\prime}\right) \varrho_{\text {rel }}\left(x^{N} ; t^{\prime}\right) d t^{\prime}= \\
= & \varrho_{\text {rel }}\left(x^{N} ; t\right)-\int_{-\infty}^{t} e^{\varepsilon\left(t^{\prime}-t\right)}\left[T_{q}\left(t, t^{\prime}\right) \frac{\partial}{\partial t^{\prime}}-\right. \\
& \left.\quad-\int_{0}^{1} T_{q}^{\tau}\left(t, t^{\prime}\right) \frac{i L_{N}}{1+(1-q)\left(t^{\prime}-t\right) i L_{N}} T_{q}^{1-\tau}\left(t, t^{\prime}\right) d \tau\right] \varrho_{\text {rel }}\left(x^{N} ; t^{\prime}\right) d t^{\prime},
\end{aligned}
$$

где

$$
T_{q}\left(t, t^{\prime}\right)=\exp _{+}\left\{-\int_{t^{\prime}}^{t} \frac{i L_{N}}{1+(1-q) t^{\prime \prime} i L_{N}} d t^{\prime \prime}\right\}
$$

- параметризированный оператор эволюции. В случае $|1-q| \Omega_{\mathrm{ch}} t \ll 1$ из (36) мы получим решение уравнения типа Линдблада для $\varrho\left(x^{N} ; t\right)$. Это решение можно использовать для получения обобщенных уравнений переноса для квантовых систем. В частности, интересным является вопрос о форме кинетических уравнений для одно- и двухчастичной неравновесных функций в случае систем, которые описываются неравновесным статистическим оператором (36).

\section{5. ЗАКЛЮЧЕНИЕ}

Таким образом, мы представили обобщение метода неравновесного статистического оператора Зубарева на основе принципа максимума энтропии Реньи. Для описания кинетических процессов в газах и жидкостях, далеких от равновесия, получены неравновесный статистический оператор и обобщенные кинетические уравнения для одно- и двухчастичной функций распределения $f_{1}(x ; t)=\left\langle\hat{n}_{1}(x)\right\rangle^{t}$ и $f_{2}\left(x, x^{\prime} ; t\right)=$ $\left\langle\hat{n}_{2}\left(x, x^{\prime}\right)\right\rangle^{t}$. Мы раскрыли внутреннюю структуру обобщенных функций памяти. 
Это дало возможность показать, что кинетические уравнения содержат корреляционные функции второго и высших порядков $\Omega_{n j}, \Omega_{n F}, \Omega_{n n j}, \Omega_{n n F}, \Omega_{n n j n}, \Omega_{n n F n}$ по динамическим переменным $\hat{n}(x), \hat{\vec{j}}(x), \hat{\vec{F}}(x)$. В отличие от функций $\Omega_{\alpha \beta}$, описывающих недиссипативные процессы, диссипативные процессы в системе описываются функциями памяти кинетических уравнений $D_{\alpha \beta}$, построенными на переменных $\hat{n}(x), \hat{\vec{j}}(x), \hat{\vec{F}}(x),[1-P(t)] \hat{\vec{j}}(x)$ и $[1-P(t)] \hat{\vec{F}}(x)$.

Мы рассмотрели $q$-обобщение уравнения Лиувилля и получили его общее решение методом неравновесного статистического оператора. В случае $|1-q| \Omega_{\mathrm{ch}} t \ll 1$ можно получить решение уравнения типа Линдблада для $\varrho\left(x^{N} ; t\right)$.

Еще одна интересная задача состоит в получении $q$-обобщенных уравнений гидродинамики на основе уравнений $(24)$ и в применении их для описания процессов турбулентности и динамики фазовых переходов в жидкостях и плотных газах. Актуальной также является переформулирование представленного подхода для описания квантовых систем (в частности, квантовой турбулентности [1], [76]). Эти задачи будут рассматриваться в наших следующих работах.

\section{Список литературы}

[1] K. Yoshida, T. Arimitsu, J. Phys. A: Math. Theor., 46:33 (2013), 335501, 15 pp.

[2] P. C. Valente, J.C. Vassilicos, The non-equilibrium region of grid-generated decaying turbulence, arXiv: 1307.5898; The energy cascade in grid-generated non-equilibrium decaying turbulence, arXiv: 1307.5901.

[3] M. Tokuyama, Phys. A, 395 (2014), 31-47, arXiv: 1409.4839.

[4] P. Mendoza-Méndez, L. López-Flores, A. Vizcarra-Redón, L. F. Sánchez-Diaz, M. Medina-Noyola, Phys. A, 394 (2014), 1-16.

[5] J. Du, Phys. A, 391:4 (2012), 1718-1728.

[6] R. Guo, J. Du, Phys. A, 406 (2014), 281-286.

[7] J.P. Boon, J.F. Lutsko, C. Lutsko, Phys. Rev. E, 85:2 (2012), 021126, 7 pp., arXiv: 1110.5463.

[8] G. F. Mazenko, Phys. Rev. E, 81:6 (2010), 061102, 18 pp., arXiv: 0905.4904.

[9] G.F. Mazenko, Phys. Rev. E, 83:4 (2011), 041125, 21 pp., arXiv: 1009.3008.

[10] S. P. Das, G. F. Mazenko, J. Statist. Phys., 152:1 (2013), 159-194, arXiv: 1303.1627.

[11] P. Kostrobij, R. Tokarchuk, M. Tokarchuk, B. Markiv, Condens. Matter Phys., 17:3 (2014), 33005, 9 pp.

[12] P. A. Hlushak, M. V. Tokarchuk, Condens. Matter Phys., 17:2 (2014), 23606, 14 pp.

[13] C. A. B. Silva, A. R. Vasconcellos, J. G. Ramos, R. Luzzi, J. Statist. Phys., 143:5 (2011), 1020-1034.

[14] C. A. B. Silva, J. G. Ramos, A. R. Vasconcellos, R. Luzzi, Nonlinear higher-order hydrodynamics. Unification of kinetic and hydrodynamic approaches within a nonequilibrium statistical ensemble formalism, arXiv: 1210.7280.

[15] V. N. Tsytovich, U. de Andelis, Phys. Plasmas, 11:2 (2004), 496-506.

[16] A. I. Olemskoi, Theory of Structure Transformations in Non-equilibrium Condensed Matter, Horizons in World Physics Series, 231, NOVA Science Publ., New York, 1999.

[17] T. D. Frank, Nonlinear Fokker-Planck Equations. Fundamentals and Applications, Springer, Berlin, 2005.

[18] B. B. Markiv, R. M. Tokarchuk, P. P. Kostrobij, M. V. Tokarchuk, Phys. A, 390:5 (2011), $785-791$.

[19] J. P. Boon, S. Yip, Molecular Hydrodynamics, McGraw-Hill, New York, 1980. 
[20] И. Мрыглод, М. Токарчук, Вопросы атомной науки и техники, 3:24 (1992), 134-139.

[21] I. M. Mryglod, I. P. Omelyan, M. V. Tokarchuk, Mol. Phys., 84:2 (1995), 235-259.

[22] B. B. Markiv, I. P. Omelyan, M. V. Tokarchuk, Phys. Rev. E, 82:4 (2010), 041202, 11 pp.

[23] Д. Н. Зубарев, В. Г. Морозов, И. П. Омелян, М. В. Токарчук, ТМФ, 96:3 (1993), $325-350$.

[24] M. V. Tokarchuk, I. P. Omelyan, A. E. Kobryn, Condens. Matter Phys., 1:4(16) (1998), 687-751.

[25] A. E. Kobryn, I. P. Omelyan, M. V. Tokarchuk, J. Statist. Phys., 92:5-6 (1998), 973-994.

[26] B. Markiv, I. Omelyan, M. V. Tokarchuk, J. Statist. Phys., 155:5 (2014), 843-866, arXiv: 1308.3627.

[27] B. Markiv, M. Tokarchuk, Phys. Plasmas, 21:2 (2014), 023707, 16 pp., arXiv: 1309.2509.

[28] Д. Н. Зубарев, Неравновесная статистическая термодинамика, Наука, М., 1971.

[29] C. Cercignani, V.I. Gerasimenko, D. Ya. Petryna, Many-Particle Dynamics and Kinetic Equations, Kluwer, Dordrecht, 1997.

[30] Д. Н. Зубарев, В. Г. Морозов, Г. Рёпке, Статистическая механика неравновесных процессов, т. 1, Физматлит, М., 2002.

[31] Д. Н. Зубарев, В. Г. Морозов, Г. Рёпке, Статистическая механика неравновесных процессов, т. 2, Физматлит, М., 2002.

[32] P. P. Kostrobij, M. V. Tokarchuk, B. M. Markovych, V. V. Ignatyuk, B. V. Gnativ, Reaction-diffusion Processes in the "Metal-Gas" Systems, Publishing House of Lviv Polytechnic National University, Lviv, 2009 (in Ukrainian).

[33] C. Tsallis, J. Statist. Phys., 52:1-2 (1988), 479-487.

[34] A. Rényi, Probability Theory, Applied Mathematics and Mechanics, 10, North-Holland, Amsterdam, 1970.

[35] P. Turán (ed.), Selected Papers of Alfréd Rényi, v. 2: 1956-1961, Akadémiai Kiadó, Budapest, 1976.

[36] B. D. Sharma, D. P. Mittal, J. Math. Sci., 10 (1975), 28-40.

[37] E. Akturk, G. B. Bagci, R. Sever, Is Sharma-Mittal entropy really a step beyond Tsallis and Renyi entropies? arXiv: cond-mat/0703277.

[38] C. Beck, Contin. Mech. Thermodyn., 16:3 (2004), 293-304, arXiv: cond-mat/0303288.

[39] C. Beck, "Superstatistics: Theoretical concepts and physical applications", Anomalous Transport: Foundations and Applications, eds. R. Klages, G. Radons, I. M. Sokolov, Wiley-VCH, New York, 2008, 433-457.

[40] S. Abe, Y. Okamoto (eds.), Nonextensive Statistical Mechanics and Its Applications, Lecture Notes in Physics, 560, Springer, Heidelberg, 2001.

[41] M. Gell-Mann, C. Tsallis (eds.), Nonextensive Entropy - Interdisciplinary Applications, Oxford Univ. Press, New York, 2004.

[42] C. Tsallis (ed.), Introduction to Nonextensive Statistical Mechanics. Approaching a Complex World, Springer, New York, 2009.

[43] C. Essex, C. Schulzky, A. Franz, K. H. Hoffmann, Phys. A, 284:1-4 (2000), 299-308.

[44] J. P. Boon, J. F. Lutsko, Phys. A, 368:1 (2006), 55-62, arXiv: cond-mat/0508231.

[45] T. Arimitsu, N. Arimitsu, Phys. Rev. E, 61:3 (2000), 3237-3240.

[46] F. M. Ramos, R. R. Rosa, C. R. Neto, M. J. A. Bolzan, L. D. Abreu Sa, H. F. Campos Velho, Phys. A, 295:1-2 (2001), 250-253.

[47] J. R. Bezerra, R. Silva, J. A. S. Lima, Phys. A, 322:1-4 (2003), 256-266, arXiv: cond-mat/ 0201541.

[48] В. С. Кирчанов, ТМФ, 156:3 (2008), 444-453.

[49] Z.-H. Feng, L.-Y. Liu, Phys. A, 389:2 (2010), 237-241.

[50] J. Du, Phys. Lett. A, 329:4-5 (2004), 262-267, arXiv: cond-mat/0404602. 
[51] J. Du, Phys. A, 335:1-2 (2004), 107-114.

[52] R. M. Yulmetyev, F. M. Gafarov, D. G. Yulmetyeva, N. A. Emeljanova, Phys. A, 303:3-4 (2002), 427-438.

[53] R. M. Yulmetyev, N. A. Emelyanova, F. M. Gafarov, Phys. A, 341 (2004), 649-676.

[54] A. R. Plastino, M. Casas, A. Plastino, Phys. A, 280:3 (2000), 289-303.

[55] R. K. Niven, Chem. Eng. Sci., 61:11 (2006), 3785-3790.

[56] G. B. Bagci, Phys. A, 386:1 (2007), 79-84.

[57] A. G. Bashkirov, A. V. Vityazev, Phys. A, 277:1 (2000), 136-145.

[58] А. Г. Башкиров, ТМФ, 149:2 (2006), 299-317.

[59] P. Jizba, T. Arimitsu, Ann. Phys., 312:1 (2004), 17-59, arXiv: cond-mat/0207707.

[60] А.Г. Башкиров, А. Д. Суханов, ЖЭТФ, 122:3 (2002), 513-520.

[61] A. G. Bashkirov, Phys. A, 340:1-3 (2004), 153-162.

[62] A. S. Parvan, T.S. Biró, Phys. Lett. A, 340:5-6 (2005), 375-387.

[63] A. S. Parvan, T.S. Biró, Phys. Lett. A, 374:19-20 (2010), 1951-1957.

[64] A. Figueiredo, M. A. Amato, T. M. R. Filho, Phys. A, 367 (2006), 191-206.

[65] R. Luzzi, Á. R. Vasconcellos, J. G. Ramos, Riv. Nuovo Cimento, 30:3 (2007), 95-158.

[66] Á. R. Vasconcellos, J. G. Ramos, A. Gorenstein, M. U. Kleinke, T. G. Souza Cruz, R. Luzzi, Internat. J. Modern Phys. B, 20:28 (2006), 4821-4841.

[67] A. Vidiella-Barranco, H. Moya-Cessa, Phys. Lett. A, 279:1-2 (2001), 56-60, arXiv: quant-ph/0002071.

[68] G. J. Milburn, Phys. Rev. A, 44:9 (1991), 5401-5406.

[69] R. Bonifacio, S. Olivares, P. Tombesi, D. Vitali, Phys. Rev. A, 61:5 (2000), 053802, 8 pp., arXiv: quant-ph/9911100.

[70] G. Kaniadakis, Phys. A, 296:3 (2001), 405-425, arXiv: cond-mat/0103467.

[71] L. Borland, Phys. Rev. E, 57:6 (1998), 6634-6642.

[72] B. M. Boghosian, Bras. J. Phys., 29:1 (1999), 91-107, arXiv: cond-mat/9812154.

[73] М. Б. Менский, УФН, 173:11 (2003), 1199-1219.

[74] G. Schaller, Non-Equilibrium Master Equations, Tech. Univ., Berlin, 2014.

[75] В. В. Саргсян, З. Каноков, Г. Г. Адамян, Н. В. Антоненко, ЭЧАЯ, 41:2 (2010), 330-433.

[76] S. K. Nemirovskii, Phys. Rep., 524:3 (2013), 85-202.

[77] G. Lindblad, Commun. Math. Phys., 48:2 (1976), 119-130.

[78] G. Lindblad, Rep. Math. Phys., 10:3 (1976), 393-406.

[79] H. Dekker, Phys. Rep., 80:1 (1981), 1-110.

[80] A. Isar, A. Sandulescu, H. Scutaru, E. Stefanescu, W. Scheid, Internat. J. Modern Phys. E, 3:2 (1994), 635-714, arXiv: quant-ph/0411189. 